\title{
Efficacy and Satisfaction Rate in Postpartum Intrauterine Contraceptive Device Insertion: A Prospective Study
}

\author{
Pulwasha M. Iftikhar ${ }^{1}$, Nighat Shaheen ${ }^{2}$, Ena Arora ${ }^{3}$ \\ 1. Obstetrics and Gynecology, St. John's University, New York, USA 2. Obstetrics and Gynecology, Cantonment General \\ Hospital, Rawalpindi, PAK 3. Obstetrics and Gynecology, Icahn School of Medicine, Mount Sinai, New York, USA
}

Corresponding author: Pulwasha M. Iftikhar, pulwashamaria@gmail.com

\section{Abstract \\ Background}

Postpartum intrauterine contraceptive device (PPIUCD) reduces the rate of abortions and it is a costeffective, reversible, and convenient choice of contraception. The objective of our study was to evaluate the efficacy and satisfaction rate in women with postpartum intrauterine contraceptive device insertion.

\section{Methods}

This prospective study of immediate PPIUCD insertion was conducted at our institute from March 2016 to February 2019. Approval from the Institutional Review Board (IRB) was taken before starting the study. A total of 372 women were enrolled in the study after taking informed consent. All the women were counseled regarding different methods of contraception and birth control during antenatal checkups, early labor and immediately postpartum (within 48 hours). All the enrolled women in the study were followed for three years to determine the satisfaction and success rate of PPIUCD continuation. We also kept the record of women who discontinued PPIUCD.

\section{Results}

After the exclusion criteria, 372 women were recruited in the study. The mean gestation age at the time of delivery was 38.5 weeks with a standard deviation (SD) of 1.45. All the women were followed for short-term and long-term complications and satisfaction rates. Out of $372,51.07 \%$ of women $(n=190)$ had a spontaneous vaginal delivery, and $48.9 \%$ of women $(n=182)$ had a cesarean section but there was no significant long-term satisfaction outcome difference in both the groups. The highest success rate of the postpartum long-acting intrauterine contraceptive device was noted in patients who were counseled thoroughly in the antenatal and intrapartum period $61.5 \%$ as compared to those patients who were counseled either antenatally $42.2 \%$, intrapartum $35.4 \%$, or immediate postpartum $22.4 \%$ alone.

Received 09/05/2019

Review began 09/09/2019 Review ended 09/09/2019 Published 09/13/2019

๑) Copyright 2019 Iftikhar et al. This is an open access article distributed under the terms of the Creative Commons Attribution License CC-BY 3.0., which permits unrestricted use, distribution, and reproduction in any medium, provided the original author and source are credited.

\section{Conclusion}

PPIUCD insertion is an opportunity not to be missed. It allows women to obtain safe, long-acting, highly effective contraception while already within the medical system. More research data are needed in the literature with regard to counseling timings for PPIUCD insertion during the antenatal and postnatal period as it can affect the decision of women to prevent unplanned pregnancy. PPIUCD has one of the highest patient satisfaction rates among all the contraceptives.

Categories: Family/General Practice, Obstetrics/Gynecology, Public Health

Keywords: postpartum intrauterine contraceptive device, long-acting reversible contraception, family planning, planned pregnancy, satisfaction rate

\section{Introduction}

Postpartum family planning is the prevention of unintended and closely spaced pregnancies during the first 12 months following childbirth [1]. Unintended pregnancy is characterized by untimely and short pregnancy intervals and it can result in acute maternal complications and death of mothers and their children. In the United States, half of the pregnancies are unintended. According to “Healthy people 2020," almost 6.1 million pregnancies are unplanned and it has a direct association with negative health and economic outcomes [2]. An unplanned pregnancy can cause maternal and child morbidity and mortality. In a recent study of postpartum unintended pregnancies, $86 \%$ resulted from non-use of contraception and almost 50\% ended in induced abortions [2-4]. Using family planning (FP) to space births at least 36 months apart can avert $30 \%$ of maternal deaths and $10 \%$ of child deaths [3-4].

Insertion of an intrauterine contraceptive device (IUCD) immediately after delivery has been recommended by the World Health Organization (WHO), as one of the safe and effective methods of temporary 
contraception [5]. Postpartum intracontraceptive uterine device (PPIUCD) can be safely used in all breastfeeding women. Almost 39\% to $65 \%$ of women in the first-year postpartum have an unmet need for family planning [5-7]. Hence, providing contraception in this sensitive period is important. PPIUCD reduces the rate of abortions and it is a cost-effective, reversible, and convenient choice of contraception.

Early resumption of sexual activity coupled with early and unpredictable ovulation leads to many unwanted pregnancies in the first year postpartum. Moreover, in developing countries particularly, women who once go back home after delivery do not return for even a routine postpartum check-up, leave aside contraception. There are many reasons for not using contraception, specifically long-acting reversible contraception (LARC), including lack of awareness, non-availability of accessible family planning services, social pressure, and limitations on women's mobility mostly due to cultural or geographical factors. Thus, immediate postpartum family planning services need to be emphasized wherein the woman leaves the hospital with effective contraception in place. Institutional deliveries create a unique opportunity to offer a long-acting yet reversible method of contraception to women immediately following their childbirth. Cochrane reviews provide evidence of the safety and feasibility of Long-acting reversible contraception insertions in various settings [8-9].

Initiation of family planning at the time of birth is opportune, since few women in low-resource settings who give birth in a facility return for further care [7-8,10]. PPIUCD is an effective long-acting reversible contraceptive, which encourages women to give birth in health care facilities [11-12]. LARC has several advantages for use in the postpartum period as it is effective, coitus independent and does not interfere with breastfeeding.

Effective intervention is required at an individual, interpersonal, community and institutional levels, and policymakers should play their role in providing affordable and easily accessible methods of contraceptive to improve maternal and child health. Provision of awareness and education about different methods of contraception during the antenatal and postnatal period reduce the rate of unplanned pregnancies. Healthcare professionals should be well trained for the appropriate insertion of IUCD in the postpartum period $[8,10-11]$.

\section{Materials And Methods}

This prospective study of immediate PPIUCD insertion was conducted at our institute from March 2016 to February 2019. Approval from the Institutional Review Board (IRB) was taken before starting the study. A total of 372 women were enrolled in the study after taking informed consent. All the women were counseled regarding different methods of contraception and birth control during antenatal checkups, early labor and immediately postpartum (within 48 hours). In the enrolled patients, IUCD was inserted immediately postpartum regardless of the mode of delivery either intra-cesarean section (CS) or spontaneous vaginal delivery (SVD). Some patients took longer to make the decision, and IUCD was inserted after vaginal delivery within 48 hours. All the patients were followed up at six weeks, six months, and yearly for consecutive three years.

Exclusion criteria for IUCD included uterine abnormalities (unicornuate, bicornuate, didelphus, or septate uterus), hemoglobin $(\mathrm{Hb})$ of less than $8 \mathrm{~g} / \mathrm{dl}$, coagulation disorders, fever and infection during labor, prolong rupture of membranes $\geqslant 18$ hours, active lower genital tract infection and sexually transmitted diseases (STD), postpartum hemorrhage and manually removing the placenta.

Questionnaire about secondary postpartum hemorrhage (PPH), infection, expelled IUCD before follow-up, displaced IUD, and short- and long-term satisfaction rate was filled out. All the enrolled women in the study were followed for three years to determine the satisfaction and success rate of PPIUCD continuation. We also kept the record of women who discontinued PPIUCD.

All the data were statistically analyzed on SPSS version 25.0. Chi-square, Student $t$-test, and Mann-Whitney $\mathrm{U}$ test were used for categorical variables. For the determination of factors that affected success, logistic regression was used.

\section{Results}

After the exclusion criteria, 372 women were recruited in the study. The mean gestation age at the time of delivery was 38.5 weeks with a standard deviation (SD) of 1.45 . All the women were followed for short-term and long-term complications and satisfaction rates. Out of $372,51.07 \%$ of women $(n=190)$ had a spontaneous vaginal delivery, and $48.9 \%$ of women $(n=182)$ had a cesarean section, but there was no significant long-term satisfaction outcome difference in both the groups. Pelvic ultrasound was performed on each visit to ensure that IUCD is in situ. On six weeks postpartum visit, $2.9 \%$ women $(n=11)$ requested PPIUCD removal due to dissatisfaction and abdominal discomfort. Other short-term complications included irregular bleeding in 1.8\% $(\mathrm{n}=6)$, secondary PPH was $3.9 \%(\mathrm{n}=14)$, and infection in $2.1 \%(\mathrm{n}=7)$. PPIUCD was expelled in $4.2 \%$ patients $(n=15)$ and displaced in $0.005 \%$ patients $(n=2$; Figure 1$)$. However, there was no reported case of uterine perforation in this study. On subsequent visits, $2.1 \%(n=8)$ women requested IUCD removal due to interest in conception, menstrual irregularities, sexual discomfort, and abdominal 


\section{Cureus}

cramping.

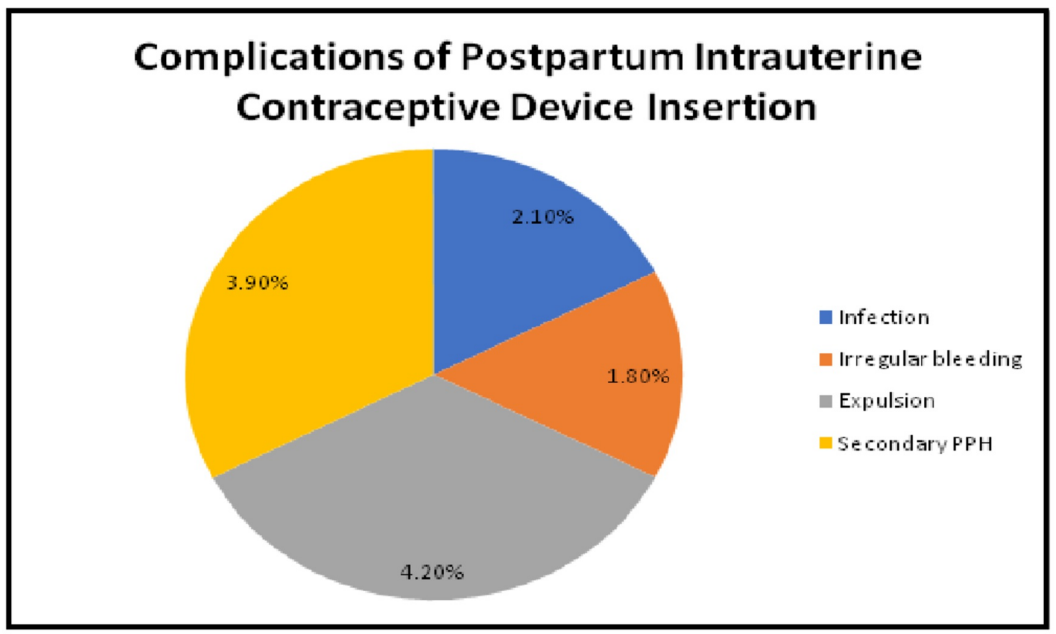

FIGURE 1: Complications of postpartum intrauterine contraceptive device

$\mathrm{PPH}$, postpartum hemorrhage

The highest satisfaction rate was observed in multiparous women between the ages of 20 to $25 \pm 5$ years. Overall, the satisfaction rate was $97.2 \%(n=350)$ at the time of insertion and $95 \%$ on the subsequent six weeks postpartum visit. Further, follow-up visits at six months and yearly for consecutive three years showed an average satisfaction rate of $93.95 \%$ (Table 1 ).

Three-year Satisfaction Rate of Postpartum Intrauterine Contraceptive Device Insertion Satisfaction rate at time of insertion

2 Satisfaction rate at six-weeks postpartum

3 Satisfaction rate at six-months postpartum

4 Satisfaction rate at one-year postpartum $93 \%$

5 Satisfaction rate at two-years postpartum

$6 \quad$ Satisfaction rate at three-years postpartum

$91 \%$

7 Overall satisfaction rate

$93.95 \%$

\section{TABLE 1: Satisfaction rate of postpartum intrauterine contraceptive device}

The highest success rate of the postpartum long-acting intrauterine contraceptive device was noted in patients who were counseled thoroughly in the antenatal and intrapartum period $61.5 \%$ as compared to those patients who were counseled either antenatally $42.2 \%$, intrapartum $35.4 \%$, or immediate postpartum $22.4 \%$ alone.

\section{Discussion}

LARC to prevent pregnancy is among the oldest and safest methods of contraception. The modern IUCD is a highly effective, safe, private, long-acting, coitus independent and rapidly reversible method of contraception with fewer side effects. Nowadays, IUCD is the most cost-effective method of contraception and many women also find it to be very convenient because it requires little attention once inserted $[3,5,8]$. The intrauterine contraceptive device is considered one of the most reliable, inexpensive, non-hormonal and reversible contraceptive methods suitable for a lactating mother because it has no negative effects on lactation and may increase its duration in some women and do not affect the quality of the breast milk. 
Across the world, particularly in developing countries, the use of long-acting reversible forms of contraceptive methods, especially PPIUCD, is being promoted largely in the postpartum period. Most women are sexually active by six weeks postpartum, and women who deliver by cesarean section may be more likely to resume sexual activity earlier than women who had vaginal deliveries [12-14]. The postpartum period is potentially an ideal time to begin contraception as women are more strongly motivated to do so at this time, which also has the advantage of being convenient for both patients and health-care providers. Other advantages of insertion of an IUCD after delivery are that the discomfort related to interval insertion can be avoided and any bleeding from insertion will be disguised by lochia [15-17].

A study by Shukla et al. reported $27.2 \%$ of women had menorrhagia after PPIUCD insertion [16]. However, in this study, the rate of secondary PPH was reported as 3.9\%. Welkovic et al. studied postpartum bleeding and infection after post placental IUCD insertion and found no difference in the incidence of excessive bleeding and infection [18].

This study was conducted to evaluate the safety and efficacy of immediate PPIUCD insertion in women delivering vaginally or by cesarean section. A total of 372 women were evaluated. The expulsion rate was found to be $4.2 \%$, which is low as compared to the other studies. From Turkey, Celen et al. reported an $11.3 \%$ cumulative expulsion rate for postpartum insertion of CuT 300B [19]. Thiery et al. from Belgium have reported a 9.4\% expulsion rate at six months for immediate post-placental insertion. The timing of insertion, counseling, and provider training are important factors for IUCD insertion in the postpartum period as quoted in the United Nations Population Information Network (UNPOPIN) report. Of these, the timing of insertion is important as it influences the risk of expulsion [20].

A research conducted in 13 countries studied infection (PID) due to IUCD and they have reported a $2.4 \%$ of infection with immediate insertion and interval insertion, which was similar to the rate of infection in our study (2.1\%). A study conducted by Ross and Winfrey in 27 countries showed that there were $65 \%$ of women who wanted to postpone the next pregnancy but they were not using any method of contraception. However, $39 \%$ of women who had delivery in the last one year had an unmet need for contraception [8-9,12,17,21].

Overall, the satisfaction rate was $97.2 \%$ at the time of insertion and $95 \%$ on the subsequent six weeks postpartum visit. By delaying birth control initiation until the six-week postpartum visit, the most vulnerable women, those who are least likely to return for their six-week postpartum visit are being placed at an increased risk for unintended pregnancies and a short pregnancy interval [16-18].

Postpartum IUCD insertion is an opportunity not to be missed and delivery may be the only time when a healthy woman comes into contact with health care providers and the chances of returning for contraceptive advice are uncertain. It allows women to obtain safe, long-acting, highly effective contraception while already within the medical system. Nowadays, PPIUCD has been established as an effective and reliable method of contraception as it offers numerous advantages: ease of insertion, minimal adverse impacts on breast-feeding, cost-effectiveness, relief of overcrowded outpatient facilities, and protection against unwanted pregnancy and consequent abortion [13-14,19].

\section{Conclusions}

PPIUCD insertion is an opportunity not to be missed. It allows women to obtain safe, long-acting, highly effective contraception while already within the medical system. Nowadays, PPIUCD has been established as an effective and reliable method of contraception as it offers numerous advantages: ease of insertion, minimal adverse impacts on breast-feeding, cost-effectiveness, relief of overcrowded outpatient facilities and protection against unwanted pregnancy and consequent abortion. More research data is required in the literature with regards to counseling timings for PPIUCD insertion during the antenatal and postnatal period as it can affect the decision of women to prevent unplanned pregnancy. PPIUCD has one of the highest patient satisfaction rates among all the contraceptives.

\section{Additional Information \\ Disclosures}

Human subjects: Consent was obtained by all participants in this study. Cantonment General Hospital issued approval Ref No. Admin/ PF/203 A. Animal subjects: All authors have confirmed that this study did not involve animal subjects or tissue. Conflicts of interest: In compliance with the ICMJE uniform disclosure form, all authors declare the following: Payment/services info: All authors have declared that no financial support was received from any organization for the submitted work. Financial relationships: All authors have declared that they have no financial relationships at present or within the previous three years with any organizations that might have an interest in the submitted work. Other relationships: All authors have declared that there are no other relationships or activities that could appear to have influenced the submitted work.

\section{References}


1. Navodani KT, Fonseka P, Goonewardena CS: Postpartum family planning: missed opportunities across the continuum of care. Ceylon Med J. 2017, 2:87-91. 10.4038/cmj.v62i2.8472

2. https://www.healthypeople.gov/2020/topics-objectives/topic/family-planning.

3. Huang YM, Merkatz R, Kang IZ: Postpartum unintended pregnancy and contraception practice among ruraltourban migrant women in Shanghai. Contraception. 2012, 86:731-738.

10.1016/j.contraception.2012.05.007

4. Report of a technical consultation on birth spacing - (2005). Accessed: September 2, 2019: https://www.who.int/maternal_child_adolescent/documents/birth_spacing05/en/.

5. Programming strategies for postpartum family planning . (2013). Accessed: September 2, 2019: https://www.who.int/reproductivehealth/publications/family_planning/ppfp_strategies/en/.

6. Black A, Guilbert E, Costescu D: Canadian contraception consensus. J Obstet Gynaecol Can. 2015, 37:936938.

7. Cleland K, Zhu H, Goldstuck N, Cheng L, Trussell J: The efficacy of intrauterine devices for emergency contraception: a systematic review of 35 years of experience. Hum Reprod. 2012, 27:1994-2000. 10.1093/humrep/des140

8. Whiteman MK, Tyler CP, Folger SG, Gaffield ME, Curtis KM: When can a woman have an intrauterine device inserted? A systematic review. Contraception. 2013, 87:666-673. 10.1016/j.contraception.2012.08.015

9. Ross JA, Winfrey WL: Contraceptive use, intention to use and unmet need during the extended postpartum period. Int Fam Plan Perspect. 2001, 27:20-7. 10.2307/2673801

10. Grimes D, Schulz K, Stanwood N: Immediate post-partum insertion of intrauterine devices . Cochrane Database Syst Rev. 2010, 5:CD003036. 10.1002/14651858.CD003036.pub2

11. Pfitzer A, Mackenzie D, Blanchard H: A facility birth can be the time to start family planning: postpartum intrauterine device experiences from six countries. Int J Gynaecol Obstet. 2015, 2:54-61. 10.1016/j.ijgo.2015.03.008

12. Pleah T, Hyjazi Y, Austin S: Increasing use of postpartum family planning and the postpartum IUD: early experiences in West and Central Africa. Glob Health Sci Pract. 2016, 2:140-52. 10.9745/GHSP-D-16-00039

13. Speroff L, Mishell DR: The postpartum visit: its time for a change in order to optimally initiate contraception. Contraception. 2008, 78:90-8. 10.1016/j.contraception.2008.04.005

14. Barrett G, Peacock J, Victor CR, Manyonda I: Caesarean section and postnatal sexual health. Birth. 2005, 32:306-311. 10.1111/j.0730-7659.2005.00388.x

15. Khajehei M, Ziyadiou S, Safari RM, Tabatabaee H: A comparison of sexual outcomes in primiparous women experiencing vaginal and caesarean births. Indian J Community Med. 2009, 34:126-30. 10.4103/09700218.51237

16. Shukla M, Qureshi S, Chandrawati: Postplacental intrauterine device insertion -- a five year experience at a tertiary care centre in north India. Indian J Med Res. 2012, 136:432-5. https://www.ncbi.nlm.nih.gov/pmc/articles/PMC3510889/

17. Kapp N, Curtis KM: Intrauterine device insertion during the postpartum period: a systematic review . Contraception. 2009, 80:327-36. 10.1016/j.contraception.2009.03.024

18. Welkovic S, Costa L, Faundes A, Ximenes R: Postpartum bleeding and infection after postplacental IUD insertion. Contraception. 2001, 63:155-8. 10.1016/S0010-7824(01)00180-9

19. Celen S, Moroy P, Suvak A, Aktulay A: Clinical outcomes of early postplacental insertion of intrauterine contraceptive devices. Contraception. 2004, 69:279-82. 10.1016/j.contraception.2003.12.004

20. Thiery M, Van KH, Van PH: The ML Cu 250; clinical experience in Belgium and Netherlands . Br J Obstet Gynecol. 1982, 89:51-3.

21. Cleland K, Zhu H, Goldstuck N, Cheng L, Trussell J: The efficacy of intrauterine devices for emergency contraception: a systematic review of 35 years of experience. Hum Reprod. 2012, 27:1994-2000. 10.1093/humrep/des140 